\title{
Hyperparasitoid volatiles as possible foraging behaviour determinants in the aphid parasitoid Aphidius uzbekistanicus (Hymenoptera: Aphidiidae)
}

\author{
Gert PETERSEN ${ }^{1}$, Christin MATTHIESEN ${ }^{1}$, Wittko FRANCKE² and URS WYSS ${ }^{1 *}$ \\ ${ }^{1}$ Institute of Phytopathology, University of Kiel, Hermann-Rodewald-Str. 9, D-24118 Kiel, Germany \\ ${ }^{2}$ Institute of Organic Chemistry, University of Hamburg, Germany
}

Key words. Foraging behaviour, Aphidiidae, Aphidius uzbekistanicus, hyperparasitoids, Alloxysta victrix, volatiles, 6-methyl-5-hepten-2-one, trans-fused iridoids, iridomyrmecin, dihydronepetalactone.

\begin{abstract}
The foraging behaviour of individual females of Aphidius uzbekistanicus Luzhetski (Hymenoptera: Aphidiidae) on oat plants with one colony of the grain aphid Sitobion avenae F. (Homoptera: Aphididae) was examined in laboratory experiments. Patch time allocation was analysed under semi-natural conditions when individual hyperparasitoid females, Alloxysta victrix Westwood (Hymenoptera: Alloxystidae) or their volatiles 6-methyl-5-hepten-2-one or a mixture of iridoid substances soaked on vermiculite dummies were present simultaneously. Patch residence times of foraging primary parasitoid females were significantly reduced in all analysed treatment groups. Attack numbers on aphids and resulting aphid mummies were also significantly reduced. The presence of hyperparasitoids or their volatiles had no influence on the proportion of time spent engaged in different behaviour by the primary parasitoid. After encounters with hyperparasitoid females or vermiculite dispensers, which had been soaked with the synthetical iridoids, A. uzbekistanicus females changed their behaviour more frequently, and they left the aphid colony at a higher rate than in the controls, where dispensers had been soaked with only pure pentane. The identification of the associated iridoid substances, produced in the hyperparasitoid mandibular glands, is briefly described and their biological significance is discussed.
\end{abstract}

\section{INTRODUCTION}

The host searching behaviour of parasitoids can be influenced by a variety of different clues (Quicke, 1997). Vet \& Dicke (1992) emphasized the important role of infochemicals as determinants for parasitoid behaviour. Chemical odours emitted from host- infested plants assist aphidiid wasps not only in locating hosts over short and long distances (Micha \& Wyss, 1995; Du et al., 1996; Micha \& Wyss, 1996; Powell et al., 1998) but also provide information about the quality of the new habitat or host.

The foraging decisions of aphid parasitoids on the plant include physiological factors such as the egg load of the searching female (Collins \& Dixon, 1986), starvation (Grasswitz \& Paine, 1993), age (Weisser, 1994) and mating status (Michaud, 1994). Factors involved in intrapatch experience of the searching females can be the colony size (Stadler \& Völkl, 1991), or encounters with hosts (Gardner \& Dixon, 1985; Cloutier \& Bauduin, 1990; Budenberg et al., 1992; Weisser, 1995; van Steenis et al., 1996) and honeydew (Bouchard \& Cloutier, 1984; Shaltiel \& Ayal, 1998). In accordance with Waage's patch-leaving decision rule (Waage, 1979), both last mentioned factors can lead to extended search in aphid colonies (Budenberg, 1990; Ahmad, 1994), whereas encounters with already parasitized hosts may lead to reduced patch residence times, as shown by van Lenteren (1991) for Leptopilina heterotoma (Thomson), a Drosophila parasitoid.
Very little is yet known about the behavioural responses of parasitic wasps in the presence of natural enemies, especially of hyperparasitoids, which can have a considerable impact on the mortality of primary parasitoids (Sullivan, 1987; Höller et al., 1993; Rosenheim, 1998; Sullivan \& Völk1, 1999). A theoretical model (Ayal \& Green, 1993) predicted that in order to maximize its own reproductive success, foraging primary parasitoids should leave patches when hyperparasitoids are present. Höller et al. (1993) supported this prediction by analysing field data. Whereas Völkl et al. (1995) did not detect any influence of three different hyperparasitoid species on two primary parasitoid species, foraging simultaneously on the same plants under laboratory conditions, Höller et al. (1994) provided first indications for a direct effect of the hyperparasitoid A. victrix on A. uzbekistanicus in Petri dish experiments and in the wind tunnels. These results could be partly reproduced in the presence of 6-methyl-5hepten-2-one (MHO), a volatile produced in the mandibular glands of the hyperparasitoid.

The aim of this study was to determine behavioural changes in the within patch foraging of the primary parasitoid A. uzbekistanicus under semi-natural conditions, using a standardized experiment, in which A.victrix females or their volatiles, MHO or recently identified trans-fused iridoids, were present simultaneously.

\footnotetext{
* Correspondence: Professor Dr. U. Wyss, Institut für Phytopathologie, Universität Kiel, Hermann-Rodewald-Str. 9, D-24118 Kiel, Germany
} 


\section{MATERIAL AND METHODS}

\section{Insect cultures}

Stock cultures of $S$. avenae were reared on oats (Avena sativa, cv. "Bruno") in a climatic chamber conditioned at a $16 \mathrm{~L}: 8 \mathrm{D}$ photoperiod, $20^{\circ} \mathrm{C}\left( \pm 1^{\circ} \mathrm{C}\right)$ and at $60-70 \%$ r.h.. Cultures of $A$. uzbekistanicus were maintained on $S$. avenae, and cultures of $A$. victrix were reared on $S$. avenae/A. uzbekistanicus under identical conditions, each in separate chambers. Mummified aphids of both, primary and secondary parasitoids were collected into plexiglass rearing tubes, where the adults could emerge. The insects were then fed with $15 \%$ sucrose before they were used for the experiment.

\section{Standardization of plants and insects}

Oats were individually sown in plastic pots $(4 \mathrm{~cm}$ diameter) and germinated at $20 \pm 1^{\circ} \mathrm{C}$. Three days before the experiments were started, 3-4 alate $S$. avenae from mass rearing were placed on the 2-3 days old seedlings to allow production of progeny. On the day of the experiment, all winged aphids and excess progeny were removed from the seedlings with fine forceps, leaving a colony of exactly 15 aphids (L1-L2) on each plant. At that date, the plant had reached a height of approximately $10-12 \mathrm{~cm}$.

Standardization of the primary parasitoids was achieved by transferring 1-4 day old mated A. uzbekistanicus females from the rearing tubes into $1 \mathrm{~cm}$ diameter plexiglass tubes containing grain aphids on oat leaves, allowing them to contact aphids for the first time. After exactly one observed sting, the females were transferred into individual gelatine capsules $\left(0.95 \mathrm{~cm}^{3}\right)$ and kept there for at least one hour before starting the experiment. This procedure was necessary since according to preliminary observations primary parasitoids without such experience would not stay on the plants. 4-10 days old A. victrix females were also individually placed in gelatine capsules prior to the experiment. Previous stinging experience was not necessary here to make them stay on the plants.

\section{Experimental design}

For each experiment, an oat plant infested with 15 aphids was placed on a turntable in an observation chamber $(51 \times 55 \times 64$ $\mathrm{cm}, \mathrm{L} \times \mathrm{W} \times \mathrm{H}$ ) that was illuminated from both sides. The experiment started as soon as the A. uzbekistanicus female left the gelatine capsule held to the plant at $1-2 \mathrm{~cm}$ height. From this moment onwards the behavior of the female was observed and recorded using the computer software "The Observer" (Noldus). By means of turning the table without vibration it was possible to monitor the foraging female at all times until it left the plant. Only those females that stayed on the plant for at least one minute were included in the analysis.

In one of the treatment groups, a female $A$. victrix was released onto the plant at the same place where the $A$. uzbekistanicus female was later released within $30 \mathrm{~s}$. The control was a sole foraging A. uzbekistanicus female without Alloxysta present. In the two other treatment groups a piece of vermiculite as dummy, serving as a dispenser, was glued to the plant at mid height using methylcellulose. Just prior to the experiment, a pentane solution of synthetic compounds (6-methyl-5-hepten2-one or a mixture of three iridoid substances) was applied in a concentration equivalent to one hyperparasitoid female. In the corresponding controls pure pentane was applied. All treatments and the controls were repeated 41 times. To avoid any possible bias due to odours remaining from previous trials, the control trials were performed on each experimental day prior to the treatment groups

The following behavioural responses of A.uzbekistanicus females were analysed: total patch residence time; number of sting attacks and mummies formed, relative time spent for different behaviours (searching, grooming, contacts with aphids and A.victrix or dummies), attack rates per minute and behavioural changes after encounters with the hyperparasitoid female or the vermiculite dummies. The behavioural records were evaluated using contingency tables $(\mathrm{K} \times \mathrm{M}$ G-test) or Mood's median test.

\section{Chemical analysis}

For chemical analysis, dissected heads of males and females were extracted with pentane (Merck, Uvasol). The concentrated extracts were analysed by coupled gas chromatography and mass-spectroscopy. A gas chromatograph Hewlett-Packard 5890 was linked to a double focussing mass spectrometer VG 70250SE. Separations were carried out by employing $30 \mathrm{~m}, 0.25$ $\mathrm{mm}$ id fused silica capillaries coated with polar (FFAP) or nonpolar (DB5) stationary phases under various temperature programs. Enantiomeric separations were executed using a $25 \mathrm{~m}$, $0.25 \mathrm{~mm}$ id fused silica capillary coated with heptakis-(6-O-tert. butyldimethylsilyl-2,3-di- $O$-methyl)- $\beta$-cyclodextrin.

\section{RESULTS}

Careful GC/MS-analyses of pentane extracts of dissected female $A$. victrix heads revealed the presence of several volatiles which proved to occur specifically in cephalic secretions. According to their mass spectra (including high resolution mass spectrometry), these compounds were identified to be oxygenated monoterpenes. Comparison of analytical data of the natural products with those of synthetic reference samples showed the major compounds of this group to represent trans-fused iridoids. By using gas chromatography on both polar and non-polar stationary phases as well as on cyclodextrins in combination with a double focussing mass spectrometer, the main component was identified to be $(4 \mathrm{~S}, 4 \mathrm{aR}, 7 \mathrm{~S}$, $7 \mathrm{aR}$ )-iridomyrmecin of approximately $95 \%$ ee accompanied by minor amounts of the diastereomer, $(4 \mathrm{~S}, 4 \mathrm{aS}, 7 \mathrm{R}$, $7 \mathrm{aS})$-iridomyrmecin. In addition, small amounts of (4R, 4aR, 7R, 7aS)-dihydronepetalactone could be identified. Traces of geranial, neral, actinidin and geranylacetone were also found to be present. The major volatile proved to be 6-methyl-5-hepten-2-one. A gas chromatogram of a

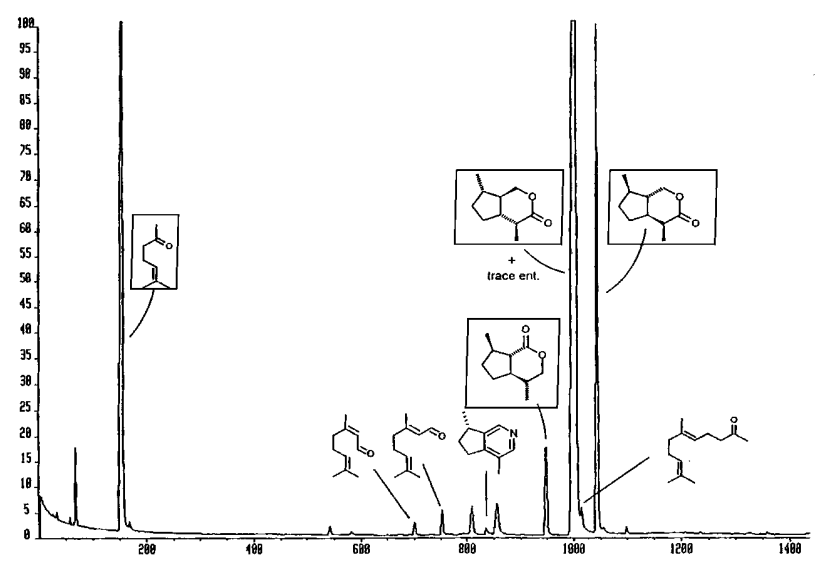

Fig. 1. Gas chromatogram of a head extract of female $A$. victrix showing the identified structures. The main volatile compounds 6-methyl-5-hepten-2-one on the left and the group of the three iridoids (marked by rectangles) were tested in our study. 
TABLE 1: Patch residence times, numbers of sting attacks of $A$. uzbekistanicus females and resulting aphid mummies in the three treatment groups and the corresponding controls. Results in the variations within a column marked with different letters differ significantly ( $\mathrm{P} \leq 0.01$, Mood's median-test).

\begin{tabular}{cccc}
\hline $\begin{array}{c}\text { Treatment } \\
\mathrm{N}=41\end{array}$ & $\begin{array}{c}\text { Patch residence } \\
\text { times in seconds } \\
\text { (means } \pm \mathrm{SD} \text { ) }\end{array}$ & $\begin{array}{c}\text { Number of } \\
\text { sting attacks } \\
\text { (means } \pm \mathrm{SD} \text { ) }\end{array}$ & $\begin{array}{c}\text { Number of } \\
\text { mummiean } \pm \mathrm{SD} \text { ) }\end{array}$ \\
\hline A. victrix & $289,4 \pm 129,9^{\mathrm{a}}$ & $4,6 \pm 6,1^{\mathrm{a}}$ & $1,2 \pm 1,1^{\mathrm{a}}$ \\
no A. victrix & $754,5 \pm 309,1^{\mathrm{b}}$ & $11,9 \pm 9,0^{\mathrm{b}}$ & $3,4 \pm 1,1^{\mathrm{b}}$ \\
MHO & $283,8 \pm 154,4^{\mathrm{a}}$ & $4,0 \pm 5,5^{\mathrm{a}}$ & $1,3 \pm 1,1^{\mathrm{a}}$ \\
Pentane control & $632,4 \pm 319,8^{\mathrm{b}}$ & $12,2 \pm 11,2^{\mathrm{b}}$ & $3,8 \pm 2,0^{\mathrm{b}}$ \\
Iridoids & $332,3 \pm 163,3^{\mathrm{a}}$ & $7,1 \pm 8,1^{\mathrm{a}}$ & $2,1 \pm 1,6^{\mathrm{a}}$ \\
Pentane control & $685,9 \pm 493,3^{\mathrm{b}}$ & $11,4 \pm 9,5^{\mathrm{b}}$ & $3,7 \pm 1,6^{\mathrm{b}}$ \\
\hline
\end{tabular}

head extract of female $A$. victrix showing the identified structures is depicted in Fig. 1. The extract of male heads looks very similar.

When the behaviour of the A. uzbekistanicus females was analysed in the presence of an $A$. victrix female or its synthetical scents, it became evident that patch residence times were highly significantly reduced when compared with the corresponding controls (Table 1). Similarly, the mean number of sting attacks and the resulting mummies from egg deposition were also significantly reduced in the treatment groups (Table 1). The mean attack rates (sting attacks per minute) did not, however, differ significantly between treatment and control groups, except for the treatment group in which $\mathrm{MHO}$ was applied to the vermiculite dummies (Fig. 2).

In all experiments the primary parasitoid females exhibited no change in the proportion of time spent in different behavioural activities as shown in Fig. 3.

Those foraging $A$. uzbekistanicus females that directly encountered the hyperparasitoids or the vermiculite dummies did that after a relatively short time span after release on the plant. A division of the overall patch residence time of these wasps into two sections before and after this first encounter, shows that the primary parasitoids remained on the plants for more than nine minutes in the control groups with pure pentane (Fig. 4). In contrast, the patch residence times were significantly reduced

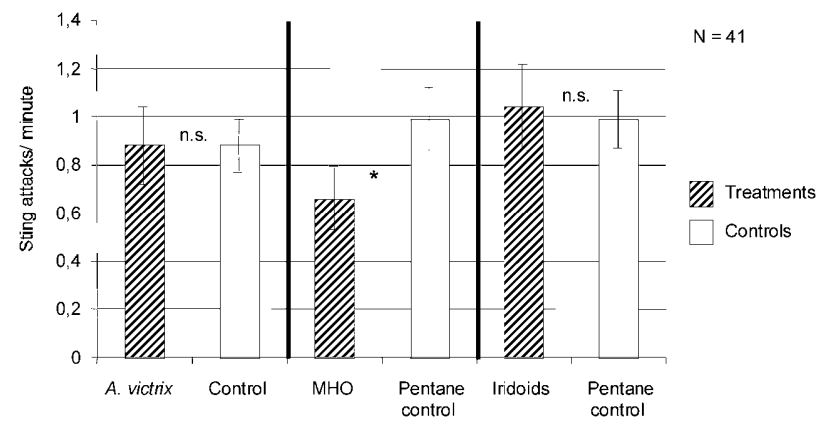

Fig. 2. Sting attack rate of $A$. uzbekistanicus females in the three treatment groups and the corresponding controls. Values in bars represent means \pm SE (n.s.: not significant; *significant $\mathrm{p} \leq 0.05$, Mood's Median Test).

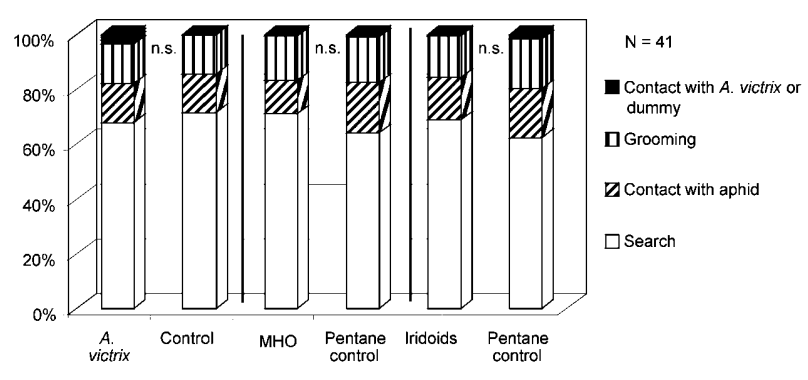

Fig. 3. Proportions of time spent by A. uzbekistanicus females for various activites in the three treatment groups and the corresponding controls. No significant differences are evident between treatment and control groups (contingency tables, G-test).

after the first encounter with the hyperparasitoid females or the vermiculite dummies soaked with synthetic MHO or iridoids (Fig. 4).

After the first encounter with an $A$. victrix female, the formerly searching $A$. uzbekistanicus females changed their behaviour in about $40 \%$ of all cases, by either grooming or leaving the plant (Fig. 5). The response occurred immediately or after a brief walking period. Aphidiids that encountered vermiculite dummies soaked with $\mathrm{MHO}$ for the first time changed their behaviour in $50 \%$ of all cases, but the proportion of encounters leading to departures was smaller than in the treatment with living hyperparasitoids. The distribution of behaviours did not differ significantly from that of the corresponding control (Fig. 5). However, when the female primary parasitoids encountered the vermiculite dummies soaked with iridoid substances for the first time, they left the plant in $20 \%$ of all cases, and they showed an overall change of behaviour of $75 \%$, which, compared to the controls, was highly significantly different (Fig. 5).

\section{DISCUSSION}

In recent years the impact of hyperparasitoids on primary parasitoids in the field has been extensively examined (for a review see Sullivan \& Völkl, 1999).

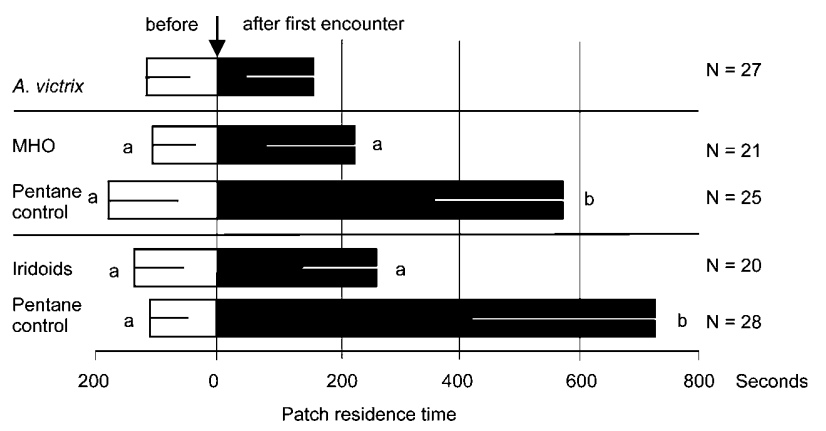

Fig. 4. Overall patch residence time of those $A$. uzbekistanicus females that encountered $A$. victrix females or the vermiculite dummies, divided into two sections before and after the first encounter. Different letters beside bars (means $\pm \mathrm{SE}$ ) indicate significant differences between treatment and control groups ( $p$ $\leq 0.01$, Mood's Median Test). 


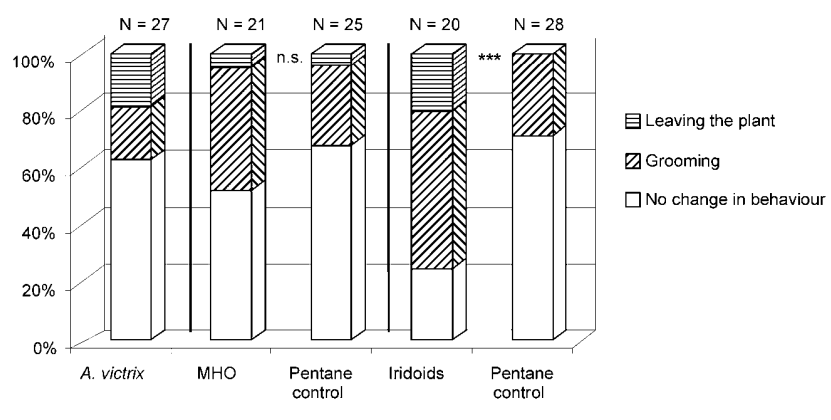

Fig. 5. Distribution of behavioural responses of searching $A$. uzbekistanicus females after the first encounter with $A$. victrix females or the vermiculite dummies within the three treatment groups and the corresponding controls. [n.s.: not significant; $* * *$ significant $\mathrm{p} \leq 0.001$, contingency tables (G-test) ].

Hyperparasitism, considered as one of the most important mortality factors of aphid primary parasitoids (Höller et al., 1993; Rosenheim, 1998), may seriously affect their biological control potential. As the reproductive success of aphid primary parasitoids is predominantly determined by their foraging decisions, hyperparasitism may have had a decisive effect on the evolution of their foraging strategies (Mackauer \& Völkl, 1993; Weisser, 1994).

Based on our results it can be concluded that the hyperparasitoid $A$. victrix exerts a certain influence on the searching behaviour of $A$. uzbekistanicus females. In the presence of the hyperparasitoid, the aphidiid wasps left the oat plants with aphid colonies significantly earlier than in the controls, which led to reduced sting attacks and numbers of offspring (Table 1). No effect could be shown concerning the proportion of time spent in different behaviour of the primary parasitoids (Fig. 3). Obvious behavioural interactions, e.g. aggression or defence, were never observed when $A$. uzbekistanicus encountered $A$. victrix and can thus be excluded as determinants for leaving reactions of the parasitoids. As special care was taken to standardize the experimental procedures, the observed behavioural responses of $A$. uzbekistanicus females can be attributed to the presence of the hyperparasitoid and its emitted volatiles, which are produced in their mandibular glands. As similar results in patch residence time were obtained when synthetic mandibular gland compounds (MHO and iridoids) were applied on vermiculite dummies (Table 1), these chemicals are most likely involved in the patch leaving reaction of A. uzbekistanicus females. A. victrix uses these volatiles for intraspecific communication (Micha et al., 1993) and for aphid hyperparasitization (Petersen et al., in preparation). Furthermore, the tested substances are also compounds of the mandibular gland secretion of several other hyperparasitoid species (Ibarra-Wiltschek, 1995; Dettner et al., preparation). This secretion functions in Alloxysta brevis (Thomson) as chemical defence against honeydew collecting ants (Völkl et al., 1994) or predating spiders (Hübner \& Dettner, 1998). The mandibular gland secretion of the hyperparasitoids is, therefore, likely to be used as kairomone by primary parasitoids to detect their enemy and to react accordingly by leaving the patch. This assumption may not be valid for other systems, since such an effect could not be shown by Völkl et al. (1995) for the primary parasitoids Aphidius funebris Mackauer or Lysiphlebus cardui (Marshall), when simultaneously searching hyperparasitoids were present.

Whereas all tested volatiles elicited reduced patch residence times in our experiments (Table 1), only the presence of MHO led to reduced sting attack rates in $A$. uzbekistanicus females (Fig. 2). This result agrees with investigations by Höller et al. (1994) and could be explained using the model of Ayal \& Green (1993), in which such a reduction of attack rates is suggested as an additional possibility for the parasitoid females to maximize their own reproductive success in combination with patch leaving when hyperparasitoids are present in high densities. Eggs deposited elsewhere would have a better chance to develop in an area with a reduced risk of being hyperparasitized. However, as the rate of sting attacks is not reduced in the presence of living $A$. victrix females, the observed effect of MHO is more likely artificial and possibly due to relatively high concentrations of $\mathrm{MHO}$ used in the experiments compared to the small amounts of mandibular gland secretion usually released by one $A$. victrix female.

MHO appears to originate from a wide range of different sources within aphid-parasitoid-hyperparasitoid systems and is, therefore, easy to detect, but it may not always provide reliable information for searching primary parasitoids. This ketone is present in their hyperparasitoid enemies but it is also emitted by aphid-infested plants, e.g. Vicia faba L., where it functions as one of the host finding cues for Aphidius ervi (Du et al., 1998). Volatiles containing $\mathrm{MHO}$, released by aphid-infested wheat plants, provide aphids (Quiroz et al., 1997) and aphid tending ants (Cordova-Yamauchi et al., 1998) information about the host habitat quality. So it seems improbable that primary parasitoids would use MHO as sole information source.

When only those $A$. uzbekistanicus females are considered that encountered the hyperparasitoid females or the vermiculite dummies soaked with one or the other synthetic substances it becomes obvious that the first direct contact with hyperparasitoids affects their patch leaving decisions (Fig. 4). However, only direct contacts with the iridoid substances elicited significant behavioural changes in the searching females such as extensive grooming or departures at high percentages (Fig. 5). The iridoids are not as volatile as MHO (Fig. 1); they may be active as semiochemicals upon contact. Iridoids are well-known as defence substances in different insects and can cause "knock down" effects (e.g. Jefson et al., 1983; Huth \& Dettner, 1990). Some ants use iridoids as an alarm pheromone, which they release when disturbed (do Nascimento et al., 1998), or as trail following pheromones (Simon \& Hefetz, 1991). The advantage of using similar chemicals that frequently occur in ants may explain why hyperparasitoids are able to exploit ant-attended aphid colonies (Völkl et al., 1994). However, most iridoids found in ants or in other insects are cis-fused with respect to their 
stereochemistry. To the best of our knowledge, this is the first time that trans-fused iridoids (Fig. 1), which are anyhow very rare in nature, have been identified in insects. Interestingly, the cyclopentanoids found as sex pheromones of aphids (Dawson et al., 1996, Hardie et al., 1997), are structurally related to one of the iridoids present in A. victrix (dihydronepetalactone), however, the aphid compounds are also cis-fused. The complete identification procedure and the syntheses of all trans-fused stereoisomers of iridomyrmecin and dihydronepetalactone will be published elsewhere.

ACKNOWLEDGEMENTS. We thank G. Mölck, D. Eder and S. Micha for comments on earlier drafts of the manuscript and $M$. Wittke for technical assistance. G.P. was supported by the Deutsche Forschungsgemeinschaft (DFG), Wy 9/16.

\section{REFERENCES}

Ayal Y. \& Green R.F. 1993: Optimal egg distribution among host patches for parasitoids subject to attack by hyperparasitoids. Am. Nat. 141: 120-138.

AHMAD M. 1994: Study of the foraging behaviour of Aphidius matricariae Haliday (Hymenoptera: Aphidiidae). Mysore $J$. Agric. Sci. 28: 127-131.

Bouchard Y. \& Cloutier C. 1984: Honeydew as a source of host-searching kairomones for the aphid parasitoid Aphidius nigripes (Hymenoptera: Aphidiidae). Can. J. Zool. 62: $1513-1520$.

Budenberg W.J. 1990: Honeydew as a contact kairomone for aphid parasitoids. Entomol. Exp. Appl. 55: 139-148.

Budenberg W.J., Powell W. \& Clark S.J. 1992: The influence of aphids and honeydew on the leaving rate of searching aphid parasitoids from wheat plants. Entomol. Exp. Appl. 63: 259-264.

Cloutier C. \& Baudun F. 1990: Searching behavior of the aphid parasitoid Aphidius nigripes (Hymenoptera: Aphidiidae) foraging on potato plants. Envir. Entomol. 19: 222-228.

Collins M.D. \& Dixon A.F.G. 1986: The effect of egg depletion on the foraging behaviour of an aphid parasitoid. J. Appl. Entomol. 102: 342-352.

Cordova-Yamauchi L., Glanoli E., Quiroz A. \& Niemeyer H.M. 1998: The Argentine ant, Linepithema humile (Hymenoptera: Formicidae: Dolichodorinae) is sensitive to semiochemicals involved in the spacing behaviour in the bird cherry-oat aphid Rhopalosiphum padi (Sternorrhyncha: Aphididae). Eur. J. Entomol. 95: 501-508.

Dawson G.W., Pickett J.A. \& Smiley D.W.M. 1996: The aphid sex pheromone cyclopentanoids: Synthesis in the elucidation of structure and biosynthetic pathways. Bioorganic Medic. Chem. 4: 351-361.

Do Nascimento R.R., Billen J., Santana A.E.G., Morgan E.D. \& Harada A.Y. 1998: Pygidial gland of Azteca nr. bicolor and Azteca chartifex: morphology and chemical identification of volatile components. J. Chem. Ecol. 24: 1629-1637.

Du Y., Poppy G. \& Powell W. 1996: Relative importance of semiochemicals from first and second trophic levels in host foraging behavior of the aphid parasitoid Aphidius ervi. $J$. Chem. Ecol. 22: 1591-1605.

Du Y., Poppy G.M., Powell W., Pickett J.A., Wadhams L.J. \& WooDCock C.M. 1998: Identification of semiochemicals released during aphid feeding that attract parasitoid Aphidius ervi. J. Chem. Ecol. 24: 1355-1368.
Gardner S.M. \& Dixon A.F.G. 1985: Plant structure and the foraging success of Aphidius rhopalosiphi (Hymenoptera: Aphidiidae). Ecol. Entomol. 10: 171-179.

Grasswitz T.R. \& PAINE T.D. 1993: Influence of physiological state and experience on the responsiveness of Lysiphlebus testaceipes (Cresson) (Hymenoptera: Aphidiidae) to aphid honeydew and to host plants. J. Insect Behav. 6: 511-528.

Hardie J., Peace L., Pickett J.A., Smiley D.W.M., Storer I.R. \& WaDHAMS L.J. 1997: Sex pheromone stereochemistry and purity affect field catches of male aphids. J. Chem. Ecol. 23: 2547-2554.

Höller C., Borgemeister C., Haardt H. \& Powell W. 1993: The relationship between primary parasitoids and hyperparasitoids of cereal aphids: an analysis of field data. J. Anim. Ecol. 62: 12-21.

Höller C., Micha S.G., Schulz S., Francke W. \& Pickett J.A. 1994: Enemy-induced dispersal in a parasitic wasp. Experientia 50: 182-185.

HứBner G. \& Dettner K. 1998: Hyperparasitoiden vs. Spinnen: Wie wirkt das Alloxystiden-Mandibeldrüsensekret auf Breitbandprädatoren? DGaaE Nachr. 12: 89-90.

Huth A. \& Dettner K. 1990: Defense chemicals from abdominal glands of 13 rove beetle species of subtribe Staphylinina (Coleoptera: Staphylinidae, Staphylininae). J. Chem. Ecol. 16: 2691-2711.

IBARRA-WILTSCHEK D. 1995: Identifizierung und Synthese monound sesquiterpenoider Inhaltsstoffe aus Hymenopteren. $\mathrm{PhD}$ Thesis, Univ. Hamburg, Germany, 190 pp.

Jefson M., Meinwald J., Nowicki S., Hicks K. \& Eisner T. 1983: Chemical defence of a rove beetle (Creophilus maxillosus). J. Chem. Ecol. 9: 159-180.

MaCKaUer M. \& VÖLKL W. 1993: Regulation of aphid populations by aphidiid wasps: Does parasitoid foraging behaviour or hyperparasitism limit impact? Oecologia 94: 339-350.

MichA S.G. \& Wyss U. 1995: Bedeutung von Pflanzenduftstoffen bei der Wirtssuche von Aphidius uzbekistanicus (Hymenoptera, Aphidiidae), eines Parasitoiden der Großen Getreideblattlaus (Sitobion avenae). Gesunde Pfl. 47: 300-307.

Micha S.G. \& Wyss U. 1996: Aphid alarm pheromone (E)-ßfarnesene: a host finding kairomone for the aphid primary parasitoid Aphidius uzbekistanicus (Hymenoptera, Aphidiinae). Chemoecology 7: 132-139.

Michaud J.P. 1994: Differences in foraging behaviour between virgin and mated aphid parasitoids (Hymenoptera: Aphidiidae). Can. J. Zool. 72: 1597-1602.

Powell W., Pennacchio F., Poppy G.M. \& Tremblay E. 1998: Strategies involved in the location of hosts by the parsitoid Aphidius ervi Haliday (Hymenoptera: Braconidae: Aphidiinae). Biol. Control 11: 104-112.

Quicke D.L.J. 1997: Parasitic Wasps. Chapman \& Hall, Cambridge, UK, $470 \mathrm{pp}$.

Qurroz A., Petterson J., Pickett J.A., Wadhams L.J. \& NieMEYER H.M. 1997: Semiochemicals mediating spacing behaviour of cherry-oat aphid, Rhopalosiphum padi feeding on cereals. J. Chem. Ecol. 23: 2599-2607.

RosENHEIM J.A. 1998: Higher-order predators and the regulation of insect herbivore populations. Annu. Rev. Entomol. 43: $421-447$.

Shaltiel L. \& Ayal Y. 1998:The use of kairomones for foraging decisions by an aphid parasitoid in small host aggregations. Ecol. Entomol. 23: 319-329.

Simon T. \& Hefetz A. 1991: Trail-following responses of Tapinoma simrothi (Formicidae: Dolichoderinae) to pygidial gland extracts. Insectes Soc. 38: 17-25. 
STADLER B. \& VölKL W. 1991: Foraging patterns of two aphid parasitoids, Lysiphlebus testaceipes and Aphidius colemani on banana. Entomol. Exp. Appl. 58: 221-229.

Sullivan D.J. 1987: Insect hyperparasitism. Annu. Rev. Entomol. 32: 49-70.

Sullivan D.J. \& VöLKL W. 1999: Hyperparasitism: Multitrophic ecology and behavior. Annu. Rev. Entomol. 44: 291-315.

Van Steenis M.J., El Khawass K.A.M.H., Hemerik L. \& VaN LENTEREN J.C. 1996: Time allocation of the parasitoid Aphidius colemani (Hymenoptera: Aphidiidae) foraging for Aphis gossypii (Homoptera: Aphidae) on cucumber leaves. $J$. Insect Behav. 9: 283-295.

VAN LENTEREN J.C. 1991: Encounters with parasitized hosts: to leave or not to leave a patch. Neth. J. Zool. 41: 144-157.

VET L.E.M. \& DiCKe M. 1992: Ecology of infochemical use by natural enemies in a tritrophic context. Annu. Rev. Entomol. 37: 141-172.

VÖLKL W., HưbNer G. \& DetTNer K. 1994: Interactions between Alloxysta brevis (Hymenoptera: Cynipoidea, Alloxystidae) and honeydew collecting ants: how an aphid hyperparasitoid overcomes ant aggression by chemical defense. J. Chem. Ecol. 20: 2901-2915.

VölkL W., Kranz P., Weisser W.W. \& Hübner G. 1995: Patch time allocation and resource exploitation in aphid primary parasitoids and hyperparasitoids searching simultaneously within aphid colonies. J. Appl. Entomol. 119: 399-404.

WAAGE J.K. 1979: Foraging for patchily-distributed hosts by the parasitoid, Nemeritis canescens. J. Anim. Ecol. 48: 353-371.

WeIsser W.W. 1994: Age-dependent foraging behaviour and host-instar preference of the aphid parasitoid Lysiphlebus cardui. Entomol. Exp. Appl. 70: 1-10.

WEISSER W.W. 1995: Within-patch foraging behaviour of the aphid parasitoid Aphidius funebris: plant architecture, host behaviour, and individual variation. Entomol. Exp. Appl. 76: 133-141.

Received January 1, 2000; accepted June 5, 2000 